\title{
Enfin, un antiglucocorticoïde
}

\section{$\longrightarrow$ (suite de la page précédente)}

pricte mais dont tous les gènes potentiellement pathogènes ont été excisés, devient un élément idéal pour obtenir avec une grande efficacité de tels transferts d'information génétique.

L'obstacle majeur reste cependant que l'intégration du matériel étranger se fait au hasard et non au niveau de la région de l'ADN qu'il s'agit de remplacer (ou dont il s'agit de pallier le dysfonctionnement), si bien que l'on peut craindre que l'expression de ce gène greffé ne soit pas correcte, quantitativement ou (et) qualitativement. La description récente par Smithies el al. [1] de l'intégration précise d'un gène de globine au niveau du locus réel du gène endogène est donc extrêmement importante sur le plan théorique.

La propriété à la base de cette " recombinaison homologue " est en fait la même que celle qui implique les recombinaisons in vivo entre chromatides sœurs (crossing over); il s'agit du réarrangement spécifique entre deux brins d'ADN identiques ou sculement très proches qui se "reconnaissent" du fait de leur complémentarité.

Il reste que la fréquence avec laquelle les auteurs ont obtenu une telle intégration d'un gène étranger au niveau de son site correct reste extrêmement faible et ne saurait être encore opérationnelle. La démonstration qu'une telle approche est possible n'en constitue pas moins une date importante dans les efforts qui conduiront un jour à cette " chirurgie du gène", efficace et précise, espoir et but des généticiens moléculaires intéressés aux problèmes de pathologie humaine.

A.K.

1. Smithics O, Grecg A G, Boggs S S, et al. Inscrtion of DNA sequences into the human chromosonal B glohin locus by homologous recombination. Nalure $1985 ; 317: 230-4$.

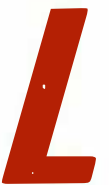

e temps des antihormones est arrivé. Parmi celles-ci, se trouvent les antagonistes des hormones stéroïdiennes èt des hormones peptidiques, agissant au niveau des récepteurs. Ainsi la spironolactone, antagoniste de l'aldostérone, a été la première antihormone utilisée. Puis sont venus les anti-cestrogènes, les anti-androgènes et bientôt les antagonistes de l'effet antidiurétique et/ou presseur de la vasopressine. On a pendant longtemps cherché des stéroides qui exercent un effet antiglucocorticoide : les produits testés avaient une activité antiglucocorticoïde in vitro, sans effet agoniste, mais perdaient tout ou partie de leur activité in vivo, se comportant comme des antagonistes partiels, seulement dans certains tests et $\grave{a}$ des doses très élevées. C'est le mérite de Philibert et al. d'avoir synthétisé dès I98I le $R U_{38486}$ ou $R U_{486}$, un I9 nor-stéroide avec une substitution en position II $\beta$ qui confere la propriété antiglucocorticoïde; ce produit a une haute affinité pour le récepteur glucocorticoïde (et également pour celui de la progestérone); il exerce des effets antiglucocorticoides in vivo chez l'animal $[\mathrm{I}]$ et chez l'homme [2,3].

Bertagna et al. [2] et Gaillard et al. [3] ont montré qu'une dose unique de $R U_{486}$ administrée à minuit ou à 2 h du matin entraîne une élévation de l' $A C T H$, de la $\beta$-lipotropine (LPH) et du cortisol plasmatiques. L'antiglucocorticoïde supprime l'effet inhibiteur que les glucocorticoïdes exercent normalement sur la sécrétion hypophysaire d'ACTH et de LPH. L'heure d'administration doit être bien choisie pour démontrer cet effet : la concentration du $R U 486$ doit être maximale quand l'antéhypophyse commence à sécréter de l'ACTH, au début de la matinée, et que la concentration plasmatique de cortisol s'élève.

Plus récemment, Nieman et al. [4] ont montré que le $R U_{4} 86$ pouvait exercer des effets cliniques bénéfiques en cas d'hypercorticisme. Ainsi, le RU 486 a été administré à des doses croissantes et fortes (jusqu'à $20 \mathrm{mg} / \mathrm{kg}$ par jour), pendant Io semaines, à un homme ayant un hypercorticisme secondaire à une sécrétion ectopique d'ACTH par une tumeur carcinoïde thoracique. Les anomalies en rapport avec l'excès de glucocorticoïdes se sont progressivement effacées au cours du traitement. L'aspect de "buffalo neck" a régressé, l'hypertension artérielle a disparu, l'alcalose et l'hypokaliémie se sont corrigées, l'état psychique s'est amélioré, le trouble de la glycorégulation s'est effacé, les anomalies hormonales habituelles au cours de l'hypercortisolisme, comme l'abaissement des taux plasmatiques de TSH, LH et testostérone, ont disparu (alors que le cortisol plasmatique et urinaire s'est élevé, comme on pouvait s'y attendre). Aucun effet secondaire n'est apparu. Ce résultat ne signifie pas que le $R U_{486}$ exercera les mêmes effets bénéfiques dans tous les hypercorticismes : dans la maladie de Cushing, on peut même craindre une libération excessive d'ACTH et l'aggravation de la maladie. Cependant ce produit représente un outil pharmacologique intéressant et peut-être un moyen thérapeutique chez certains malades.

J.P.G.

I. Gagne D, Pons M, Philibert D. RU 38486: a potent antiglucocorticoid in vitro and in vivo. 7 Steroid Biochem 1985; $23: 247-51$.

2. Bertagna X, Bertagna $C$, Luton JP, et al. The new steroid analog R U 486 inhibits glucocorticoid action in man. 3 Clin Endocrinol Metab 1984; $59: 25-8$.

3. Gaillard RC, Riondel A, Muller AF, et al. RU 486 : a steroid with antiglucocorticosteroid activity that only disinhibits the human pituitary-adrenal system at a specific time of day. Proc Natl Acad Sci USA 1984; 81 : 3879-82.

4. Nieman LK, Chrousos GP, Kellner C, et al. Successful treatment of Cushing's syndrome with the glucocorticoid antagonist RU 486. 7 Clin Endocrinol Metab 1985; 6I : 536-40. 